\title{
Infarto de miocardio con arterias coronarias no obstruidas en pediatría: una afección poco conocida
}

\author{
Myocardial infarction with unobstructed coronary arteries in pediatrics: \\ a little-known entity
}

\author{
Daniela Cleves ${ }^{1 *}$, José F. Potosi ${ }^{1}$, Jaiber A. Gutiérrez ${ }^{2}$, Walter Mosquera ${ }^{2}$ y Luis E. Ponce ${ }^{2}$ \\ ${ }^{1}$ Facultad Ciencias de la Salud, Universidad Icesi-Fundación Valle de Lili; ${ }^{2}$ Departamento de Cardiología Pediátrica, Fundación Valle de Lili. Cali, \\ Colombia
}

\begin{abstract}
Resumen
El infarto de miocardio con arterias coronarias no obstruidas (MINOCA, por sus siglas en inglés) ha ganado importancia en los últimos 20 años gracias a la dilucidación de etiologías fisiopatológicas diferentes de las causas obstructivas del flujo coronario. Diversos estudios han evidenciado una prevalencia variable, la cual es más alta en las mujeres. Se han descrito dos grupos de alteraciones en la reactividad coronaria que afectan el flujo: las causas epicárdicas y las causas microvasculares. El diagnóstico de MINOCA es de exclusión; por lo tanto, inicialmente se deben descartar otras posibles causas de isquemia, como miocarditis, miocardiopatía séptica, choque hipovolémico por trauma o quemaduras, y enfermedades renales o pulmonares. Los reportes y estudios de esta enfermedad suelen incluir pacientes adultos o mayores de 18 años. Se presenta el caso de una paciente de 16 años con antecedente de tetralogía de Fallot corregida en etapa de lactante menor y reemplazo valvular pulmonar con bioprótesis y ampliación del tronco pulmonar a los 11 años, quien ingresó con dolor torácico de características coronarias. Cursó con un diagnóstico de MINOCA por exclusión en una institución de cuarto nivel en Cali, Colombia. El diagnóstico de MINOCA en edad pediátrica es raro; sin embargo, es importante saber acerca de su existencia para brindar a los pacientes el mejor manejo disponible, de manera que se aseguren los mejores desenlaces a largo plazo.
\end{abstract}

Palabras clave: MINOCA. Infarto. No obstructivo. Reactividad coronaria. Pediatría.

\begin{abstract}
Myocardial infarction with non-obstructed coronary arteries (MINOCA) has gained importance in the last 20 years, due to the elucidation of physiopathological etiologies different from the obstructive causes of coronary flow. Different studies have shown variable prevalence, being higher in women. Different causes have been evidenced in the studies found in two groups of alterations in coronary reactivity: epicardial causes and microvascular causes. The diagnosis of MINOCA must be a diagnosis of exclusion. Therefore, other possible causes of ischemia, such as myocarditis, septic cardiomyopathy, hypovolemic shock due to trauma or burns, renal or pulmonary diseases, should be ruled out initially. The reports and studies done around this pathology usually include adult patients and people older than 18 years. We present the case of a 16-year-old patient with a
\end{abstract}

\section{Correspondencia:}

*Daniela Cleves

E-mail: clevesdaniela@ hotmail.com
Disponible en internet: 10-06-2021 Rev Colomb Cardiol. 2021;28(2):185-188 www.rccardiologia.com 0120-5633 / C 2020 Sociedad Colombiana de Cardiología y Cirugía Cardiovascular. Publicado por Permanyer. Este es un artículo open access bajo la licencia CC BY-NC-ND (http://creativecommons.org/licenses/by-nc-nd/4.0/). 
history of Tetralogy of Fallot corrected as an infant and pulmonary valve replacement with bioprothesis and enlargement of the pulmonary trunk at 11 years of age who was admitted with chest pain of coronary characteristics. The diagnosis after multiples studies and exclusion of other causes was MINOCA in a fourth level institution in Cali, Colombia.

Key words: MINOCA. Infarction. Coronary reactivity. Pediatrics.

\section{Introducción}

El infarto de miocardio con arterias coronarias no obstruidas (MINOCA, por sus siglas en inglés) ha ganado importancia en los últimos 20 años debido a la dilucidación de etiologías fisiopatológicas diferentes de las causas obstructivas del flujo coronario (obstrucción definida como estenosis $<50 \%)^{1}$. Diversos estudios han evidenciado una prevalencia que varía entre el $6 \%$ y el $25 \%$, la cual llega a ser más alta en las mujeres $(10-25 \%)^{1-3}$.

Entre las múltiples causas que se han evidenciado en los estudios se encuentran diferentes grupos de alteraciones en la reactividad coronaria: las epicárdicas y las microvasculares ${ }^{1,3-5}$, entre las cuales se incluyen espasmo coronario, disrupción de la placa aterosclerótica y disección coronaria, enfermedad de takotsubo, miocarditis y tromboembolia coronaria ${ }^{1,4}$.

El diagnóstico de MINOCA debe ser de exclusión; por tanto, inicialmente hay que descartar otras posibles causas de isquemia, como miocarditis, miocardiopatía séptica, choque hipovolémico por trauma o quemaduras, y enfermedades renales o pulmonares ${ }^{1}$. Para ello deben utilizarse todas las herramientas diagnósticas, incluidas la ecocardiografía y la arteriografía coronaria, con el fin de visualizar posibles alteraciones anatómicas que expliquen la angina'.

Los reportes y estudios sobre esta enfermedad suelen incluir pacientes adultos y personas mayores de 18 años. Se presenta el caso de una paciente de 16 años con diagnóstico de MINOCA emitido en una institución de cuarto nivel en Cali, Colombia. El consentimiento informado fue firmado por la madre de la paciente, con asentimiento de esta; adicionalmente, el reporte de caso fue aprobado por el Comité de Ética Institucional de la Fundación Valle de Lili.

\section{Caso clínico}

Paciente de sexo femenino, de 16 años, con antecedente de tetralogía de Fallot corregida en etapa de lactante menor y reemplazo valvular pulmonar con bioprótesis y ampliación del tronco pulmonar a los 11 años, en manejo con ácido acetilsalicílico (100 mg/día), furosemida (20 mg/día) y espironolactona ( $25 \mathrm{mg} / \mathrm{día})$, quien acudió al servicio de urgencias por un cuadro clínico que inició el día previo al ingreso con evento sincopal asociado a dolor torácico de tipo opresivo sin irradiación, además de disnea y mareo de varios minutos de duración.

Durante el interrogatorio refirió varios episodios similares en los meses previos, los cuales estuvieron asociados a deterioro de la clase funcional de la New York Heart Association (NYHA) I a II. Adicionalmente, en una prueba de esfuerzo se evidenció bloqueo de rama derecha, lo cual es lo esperado en pacientes con antecedente de tetralogía de Fallot corregida; sin embargo, se alcanzó un $60 \%$ de la frecuencia cardiaca máxima con realización de 5 MET y se suspendió la prueba por disnea. Fue hospitalizada para estudios de extensión.

El ecocardiograma transtorácico pediátrico no mostró hallazgos que explicaran la clínica, lo cual es de esperar si se tienen en cuenta los antecedentes de la paciente (Fig. 1).

Se realizó angiorresonancia cardiaca (Fig. 2) en la que se evidenciaron: 1) hallazgos compatibles con infarto subendocárdico que comprometía los segmentos posteriores y posterolaterales de los planos basal y medio ventricular (territorio descendente posterior; estos segmentos fueron considerados como viables, ya que tenían compromiso $<50 \%$ de transmuralidad); 2) signos de infarto del músculo papilar posterior; 3 ) leve disfunción sistólica biventricular (fracción de eyección de los ventrículos izquierdo y derecho del $49 \%$ y el $50 \%$, respectivamente); y 4) volúmenes ventriculares derechos de fin de diástole y fin de sístole con mejoría cuando se compararon con el estudio prequirúrgico. En vista de los hallazgos imagenológicos, se llevó a cateterismo cardiaco (Fig. 3) para evaluar las coronarias; en este se evidenciaron coronarias normales, estenosis de la bioprótesis pulmonar leve a moderada e insuficiencia leve, ventrículo derecho hipertrofiado, dilatación leve y función sistólica adecuada, tronco y ramas pulmonares de tamaño normal, sin defectos de contractilidad segmentaria en el ventrículo izquierdo.

Los paraclínicos realizados mostraron un perfil lipídico normal, y propéptido natriurético de tipo $\mathrm{B} \mathrm{N}$-terminal y troponina negativos. Adicionalmente, de 

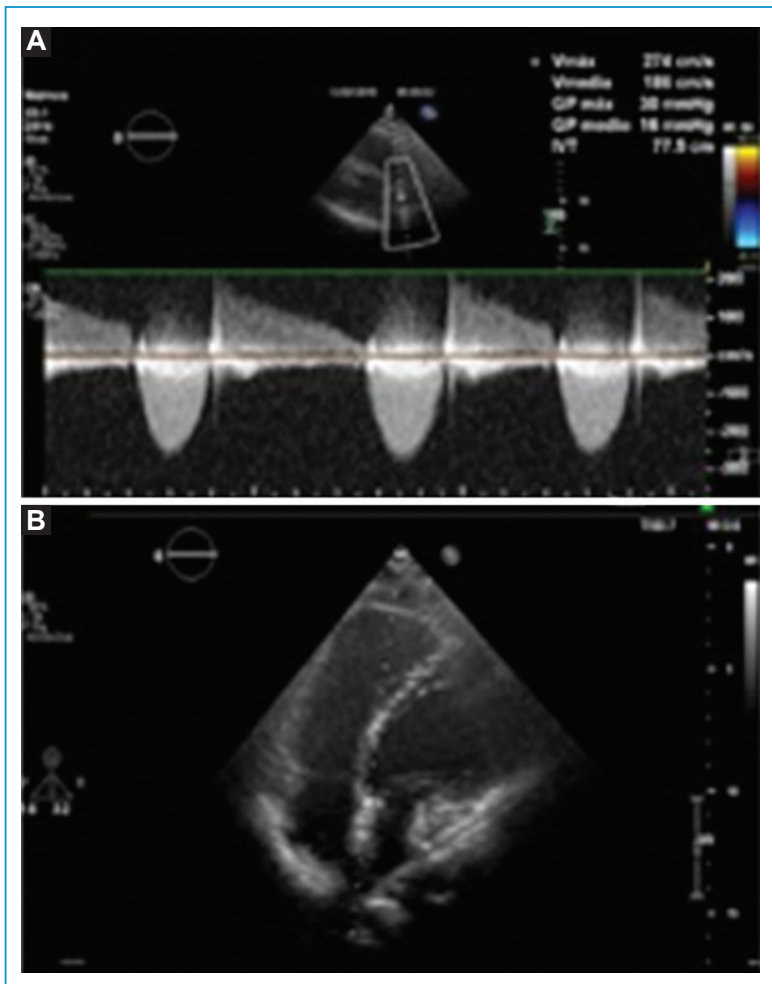

Figura 1. Ecocardiograma pediátrico transtorácico. A: tracto de salida del ventrículo derecho y la arteria pulmonar. Gradiente a través de bioprótesis pulmonar. B: vista apical de cuatro cámaras. Dilatación de cámaras derechas.

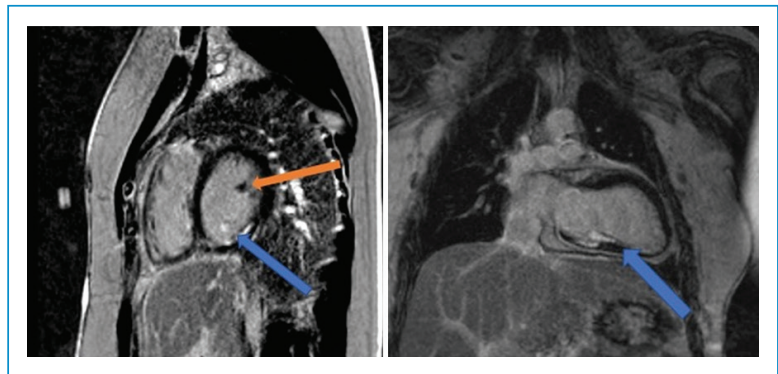

Figura 2. Angiorresonancia cardiaca con evidencia de infarto en el músculo papilar posterior (azul). El músculo papilar anterior sin infarto (naranja).

acuerdo con el sexo y la edad de la paciente, se consideró necesario descartar otras posibles causas reumatológicas, como vasculitis de pequeños vasos (anticuerpos anticitoplasma de neutrófilos [ANCA]) y síndrome antifosfolípido, que podrían generar cambios en la microvasculatura cardiaca; sin embargo, los anticuerpos anticardiolipinas (IgM-IgG), anti-beta-2-glucoproteína I (IgM-lgG), P y C ANCA, ENA, ANA y el

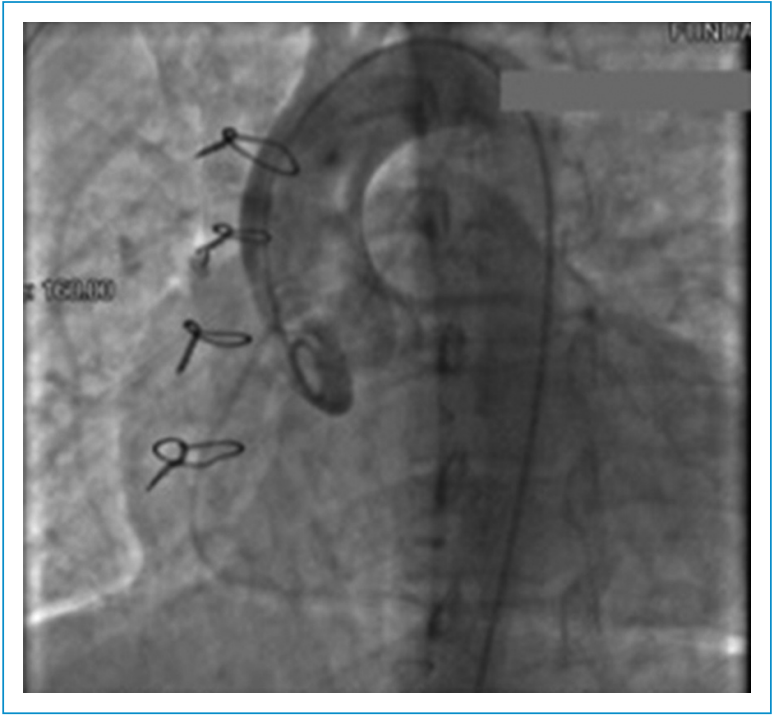

Figura 3. Angiografía coronaria. Paso normal del medio de contraste, no muestra obstrucción coronaria.

anticoagulante lúpico fueron negativos, la velocidad de sedimentación globular normal, y C3 y C4 normales.

\section{Discusión}

EI MINOCA ha ganado mayor significancia en los últimos años debido a los avances tecnológicos y de métodos diagnósticos que han permitido aclarar mecanismos fisiopatológicos diferentes de las causas obstructivas del flujo ${ }^{1,2}$. En una revisión de la literatura publicada en 2015 que incluyó 176.502 pacientes se encontró un rango de prevalencia del $1 \%$ al $14 \%$ en los diferentes estudios, con una prevalencia total del $6 \%{ }^{3}$. En comparación con mujeres con infarto agudo de miocardio secundario a obstrucción, en el grupo de MINOCA se evidenció una mayor prevalencia de mujeres jóvenes sin comorbilidad, como dislipidemia; adicionalmente, estas pacientes tienden a continuar más tiempo con angina, mientras que el $25 \%$ de las pacientes con MINOCA presenta dolor a los 12 meses posteriores al inicio del cuadro ${ }^{3}$.

Entre las múltiples causas se encuentran diferentes grupos de alteraciones en la reactividad coronaria: epicárdicas y microvasculares ${ }^{1,3-5}$. Entre las primeras se incluyen espasmo coronario, disrupción de la placa aterosclerótica y disección coronaria, mientras que en las segundas se pueden encontrar enfermedad de takotsubo, miocarditis y tromboembolia coronaria ${ }^{1,4}$. En un estudio en el que se evaluaron 224 mujeres con signos y síntomas de isquemia miocárdica sin evidencia de lesión coronaria obstructiva, las alteraciones microvasculares fueron resultado de una disminución 
a la respuesta de acetilcolina y de que los procesos de disminución de la reserva de flujo coronario se asociaron con peores desenlaces desde el punto de vista cardiovascular ${ }^{5}$.

El diagnóstico de MINOCA debe ser de exclusión; por lo tanto, hay que descartar otras posibles causas de isquemia, como miocarditis, miocardiopatía séptica, choque hipovolémico por trauma o quemaduras, y enfermedades renales o pulmonares ${ }^{1}$. Deben utilizarse para tal fin todas las herramientas diagnósticas, incluyendo la ecocardiografía y la arteriografía coronaria, para visualizar posibles alteraciones anatómicas que expliquen la angina ${ }^{1}$. Adicionalmente, existen otros estudios imagenológicos, como la angiorresonancia magnética de corazón y la tomografía computarizada cardiaca, que permiten visualizar la localización y el área de miocardio afectado, y detectar el tejido infartado o fibrótico, así como el tejido inflamado o edematoso y las anormalidades de la pared ${ }^{1}$. No obstante, es preciso tener en cuenta que el diagnóstico se dificulta cuando no se tienen los recursos disponibles.

Los reportes y estudios sobre esta enfermedad suelen incluir pacientes adultos y no hemos encontrado ninguno en población pediátrica ni en pacientes con antecedente de cardiopatía congénita. VIRGO, un estudio de caracterización en personas jóvenes con MI$\mathrm{NOCA}^{6}$, solo incluyó pacientes mayores de 18 años $^{6} \mathrm{y}$ encontró que, de los 2690 pacientes con infarto agudo de miocardio, el $11 \%$ tenían MINOCA ${ }^{6}$. Adicionalmente, se evidenció que las mujeres tenían más riesgo de MINOCA que los hombres (odds ratio $=4.84$; intervalo de confianza: 3.29-7.13) y que los pacientes con diagnóstico de MINOCA tenían menos probabilidad de tener factores de riesgo cardiovascular tradicionales (8.7 vs. $1.3 \% ; P<0.001$ ), pero presentaban más predisposición a estados de hipercoagulabilidad que los pacientes con enfermedad coronaria obstructiva ${ }^{6}$.

Una de las fortalezas del caso presentado es que se trata de una afección poco reconocida en la población pediátrica, ya que la mayoría de los reportes corresponden a adultos. Adicionalmente, se trata de un caso que recibió manejo en una institución de cuarto nivel, por lo que se pudo realizar seguimiento imagenológico y paraclínico para descartar otras causas. Una de las debilidades del caso es que el seguimiento a largo plazo se dificulta por problemas administrativos con las diferentes entidades de salud.

\section{Conclusiones}

EI MINOCA ha ganado fuerza en los círculos de cardiología en los últimos años para explicar patologías coronarias no obstructivas sin causa aparente. Existe poca información acerca del MINOCA en población pediátrica, pero como demuestra este caso, existe y no debe pasar inadvertido. Consideramos que es un tema importante que requiere mayor investigación y más atención por parte del personal médico, para así brindar el mejor cuidado posible a los pacientes y encontrar métodos que ayuden a predecir aquellos que están en riesgo de presentar esta patología.

\section{Conflicto de intereses}

Los autores no declaran conflicto de intereses.

\section{Responsabilidades éticas}

Protección de personas y animales. Los autores declaran que para esta investigación no se han realizado experimentos en seres humanos ni en animales.

Confidencialidad de los datos. Los autores declaran que han seguido los protocolos de su centro de trabajo sobre la publicación de datos de pacientes.

Derecho a la privacidad y consentimiento informado. Los autores han obtenido el consentimiento informado de los pacientes y/o sujetos referidos en el artículo. Este documento obra en poder del autor de correspondencia.

\section{Bibliografía}

1. Zuluaga-Quintero M, Cano-Granda CC. Infarto agudo de miocardio sin enfermedad coronaria ateroesclerótica obstructiva. latreia. 2018;31:371-9. DOI: 10.17533/udea.iatreia.v31n4a04.

2. Poku N, Noble S. Myocardial infarction with non-obstructive coronary arteries (MINOCA): a whole new ball game. Expert Rev Cardiovasc Ther. 2017;15:7-14. DOI: 10.1080 / 14779072.2017.1266256.

3. Pasupathy S, Air T, Dreyer RP, Tavella R, Beltrame JF. Systematic review of patients presenting with suspected myocardial infarction and non-obstructive coronary arteries (MINOCA). Circulation. 2015;137:86170. DOI: 10.1161/CIRCULATIONAHA.114.011201.

4. Niccoli G, Scalone G, Crea F. Acute myocardial infarction with non-obstructive coronary atherosclerosis: mechanisms and management. Eur Heart J. 2015;36:475-81. DOI: 10.1093/eurheartj/ehu469.

5. AlBadri A, Bairey Merz CN, Johnson BD, Wei J, Mehta PK, Cook-Wiens G. Impact of abnormal coronary reactivity on long-term clinical outcomes in Women. J Am Coll Cardiol. 2019;73:684-93. DOI: 10.1016/j. jacc.2018.11.040.

6. Safdar B, Spatz ES, Dreyer RP, Beltrame JF, Lichtman JH, Spertus JA, et al. Presentation, clinical profile, and prognosis of young patients with myocardial infarction with nonobstructive coronary arteries (MINOCA): results from the VIRGO study. J Am Heart Assoc. 2018;7:e009174. 